\title{
Implementation of the mechanistic wall boiling model in IC engine cooling gallery simulation
}

\author{
Oleg Abyzov ${ }^{1, *}$, Yuri Galyshev ${ }^{1}$, and Andrey Metelev ${ }^{1}$ \\ ${ }^{1}$ Peter the Great St. Petersburg Polytechnic University, Polytechnicheskaya 29, St. Petersburg, \\ 195251, Russian Federation
}

\begin{abstract}
The ever increasing power rates of modern IC engines pose a great challenge for maintaining the optimal temperature conditions. The current trend in the thermal management of IC engines is to utilize the limited and controlled nucleate boiling to achieve higher heat transfer rates. The modern CFD instrumentation offers a wide range of methods for predicting nucleate and film boiling. Of the available models the mechanistic RPI wall boiling model is selected in present paper in the cojuntion with the Eulerian multiphase model available in ANSYS Fluent solver to simulate subcooled nucleate boiling in engine cooling jacket.Results obtained from the simulation of boiling flow in cooling passages is then compared with the experimental data from author's previous work and show a decent agreement.
\end{abstract}

\section{Introduction}

Cooling plays an important role in maintaining engine performance under severe mechanical and thermal conditions. Modern heavy-duty engines need more efficient cooling systems, which is achieved, along with other measures, through development of more optimized designs of cooling jackets, allowing increased flowrates in critical zones where higher temperatures may occur [1]. Another way to increase cooling rate is by utilizing the effect of subcooled nucleate boiling. The boiling taking place in the engine cooling passage is considered as subcooled, since the bulk temperature of the fluid is normally controlled below the saturation point. Effective use of boiling in engine coolant design can make the structure more compact.

In the past years CFD has made a significant progress in the analysis of fluid flows in complex systems including the engine cooling jackets. Various studies have been performed to gain insight into the different heat transfer mechanisms such as the boiling phenomena. The results of these studies are different approaches to represent the effect of boiling in the overall heat transfer, which include:

- Direct numerical simulation (DNS) for nucleation and bubble growth near the superheated wall.

- Boiling models

* Corresponding author: oleg.abyzov@yandex.ru 
With the growing computational powers, modern systems provide, it has become possible to perform DNS for some industrial flows [2-4]. Although due to still enormous computational costs such studies are restricted only to cases with a small number of growing bubbles in a simple geometry with ideal surfaces.

Boiling models fall into two main categories:

- Empirical models i.e. models relying on general empirical correlations that relate the total wall heat transfer rates or heat transfer coefficients with wall superheat [5-7].

- Mechanistic models or partitioning models that try to break down and describe or mimic certain heat transfer mechanisms [8-14]. Mechanistic models have an advantage over fully empirical models that they can be extended to make predictions outside of the range of specific conditions in certain cases that makes them more general-purpose.

Over the last two decades there has been a development of a fully mechanistic boiling model in a two-fluid multiphase framework. One of the examples, RPI wall boiling model, developed by Kurul and Podowski [15] in Rensselaer Polytechnic Institute, has been established as the modeling approach for mechanistic prediction of boiling phenomena and is used as the default boiling model in the commercial CFD solver ANSYS Fluent. Although widely used to investigate boiling in conditions found in the nuclear industry this model was given controversial reports for its applicability in low pressure conditions found in IC engine's cooling gallery [16,17]. The aim of this work is to investigate the performance of RPI boiling model in a case similar to a typical IC engine cooling jacket.

\section{Methods}

\subsection{Numerical methodology}

To properly simulate the complex process of flow boiling it is necessary to account for the contribution of vapor formation to the mean flow dynamics. To do this a fully multiphase Eulerian model was utilized in present setup in conjunction with RPI wall boiling model. The 3D fluid dynamics calculations were carried out using the ANSYS Fluent software.

\subsubsection{The Multiphase Flow Model}

The Eulerian multiphase model in ANSYS Fluent is based on Euler-Euler approach in which the different phases in the flow are treated mathematically as interpenetrating continua. It solves a set of momentum and continuity equations for each phase. Coupling is achieved through the pressure and interphase exchange coefficients.

For more information about Eulerian model please refer to Fluent Theory Guide.

\subsubsection{Turbulence model}

Standard $k-\varepsilon$ turbulence model is used in present work. Within the multiphase model the mixture form of the $k-\varepsilon$ model is often used. For more information about $k-\varepsilon$ model please refer to [18].

\subsubsection{Wall boiling model}

RPI model for nucleate boiling is used in this work. It represents total heat flux from the wall as a sum of three components: the convective heat flux, the quenching heat flux, and the evaporative heat flux 


$$
q_{w}=q_{C}+q_{Q}+q_{E}
$$

The heated wall surface is subdivided into area $A_{b}$, which is covered by nucleating bubbles and a portion $\left(1-A_{b}\right)$, which is covered by the fluid.

Enhanced convection takes place during nucleate boiling as a form of the convection in the mean flow enhanced by the flow disturbances caused by moving vapor bubbles. The convective heat flux $q_{c}$ is expressed as

$$
q_{C}=h_{C}\left(T_{w}-T_{l}\right) \cdot\left(1-A_{b}\right)
$$

where $h_{c}$ is the single phase heat transfer coefficient, and $T_{w}$ and $T_{l}$ are the wall and liquid temperatures, respectively.

The "quenching" heat flux $q_{Q}$ represents the cyclic averaged transient heat transfer process when liquid flows from the bulk liquid to the wall after a bubble moves away from the surface and is expressed as

$$
q_{Q}=\frac{2 k_{l}}{\sqrt{\pi \lambda_{l} T}}\left(T_{w}-T_{l}\right)
$$

where $k_{l}$ is the conductivity, $T$ is the periodic time, and $\lambda_{l}=\frac{k_{l}}{\rho_{l} c_{p l}}$ is the diffusivity.

The evaporative flux $q_{E}$ refers to evaporation of the thin microlayer of liquid that is left below a growing bubble and is expressed as

$$
q_{E}=V_{d} N_{w} \rho_{v} h_{f v}
$$

Where $V_{d}$ is the volume of the bubble based on the bubble departure diameter, $N_{w}$ is the active nucleate site density, $\rho_{v}$ is the vapor density, and $h_{v}$ is the latent heat of evaporation. All the mass transfer in RPI model (from wall to vapor and between phases) is derived from the respective heat transfer mechanisms.

\subsubsection{Additional sub-models and correlations for multiphase and boiling models}

The models and correlations for the following terms are given without further explanation. In the present setup they were used as defaults or as recommended by Fluent user's guide.

Models and correlations for multiphase model

- Interfacial Area. The default symmetric model is recommended for boiling flows

- Drag force. Ishii formulation is used

- Lift force. Tomiyama lift coefficient model is used

- Wall lubrication force. Antal et al. model for wall lubrication coefficient is used

- Turbulent dispersion force. Lopez de Bertodano model is used

- Turbulence interaction source terms. Troshko-Hassan model is used

- Heat Exchange Coefficient. Ranz-Marshall correlation is used

Models and correlations for boiling model

- Area influence coefficient is set according to Del Valle and Kenning's findings

- Frequency of bubble departure. Cole's formulation used

- Nucleation site density. Formulation of Lemmert and Chawla is used.

- Bubblle departure diameter. Default option of Tolubinski and Kostanchuk is set 


\subsection{Validation case}

\subsubsection{Experimental setup}

For validation of the proposed model the experimental data from our previous work [19] was taken. In that study an experimental rig was developed to simulate the cylinder head cooling process. The design of test duct used in this setup allows to simulate not only a jet flow of coolant but also a stagnant flow of coolant. Zones of low velocity flow or stagnant zones when subjected to the high thermal load are the main source of vapor formation. The boiling regime in these zones is close to the situation of pool boiling.

To model both jet and stagnant flows in one experimental setup the test duct of cylindrical shape heated from one side was used. The general layout of the test duct along with pictures of flow distribution is shown in figure 1 . The heat was conducted through 10 $\mathrm{mm}$ aluminum plate with thermocouples positioned in 3 points on both sides of the plate $(6$ total) which allowed the calculation of instantaneous local heat fluxes. The temperatures on the inlet and outlet of the channel were also measured.

Genaral test parameters were as follows:

- Inlet velocity: $1 \mathrm{~m} / \mathrm{s}$

- Inlet coolant temperature: $90^{\circ} \mathrm{C}$

- Operating pressure: 1 bar abs

- Material of test sample: Aluminium alloy

- Surface finish: smooth

- Coolant types: distilled water and 50-50 ethylen glycol antifreeze

A total of 2 tests were performed at different types of coolant. In both tests the wall temperature was measured at coolant side and heated side of the test sample. Four experimental curves of wall heat flux versus wall temperature were then constructed based on measured data.

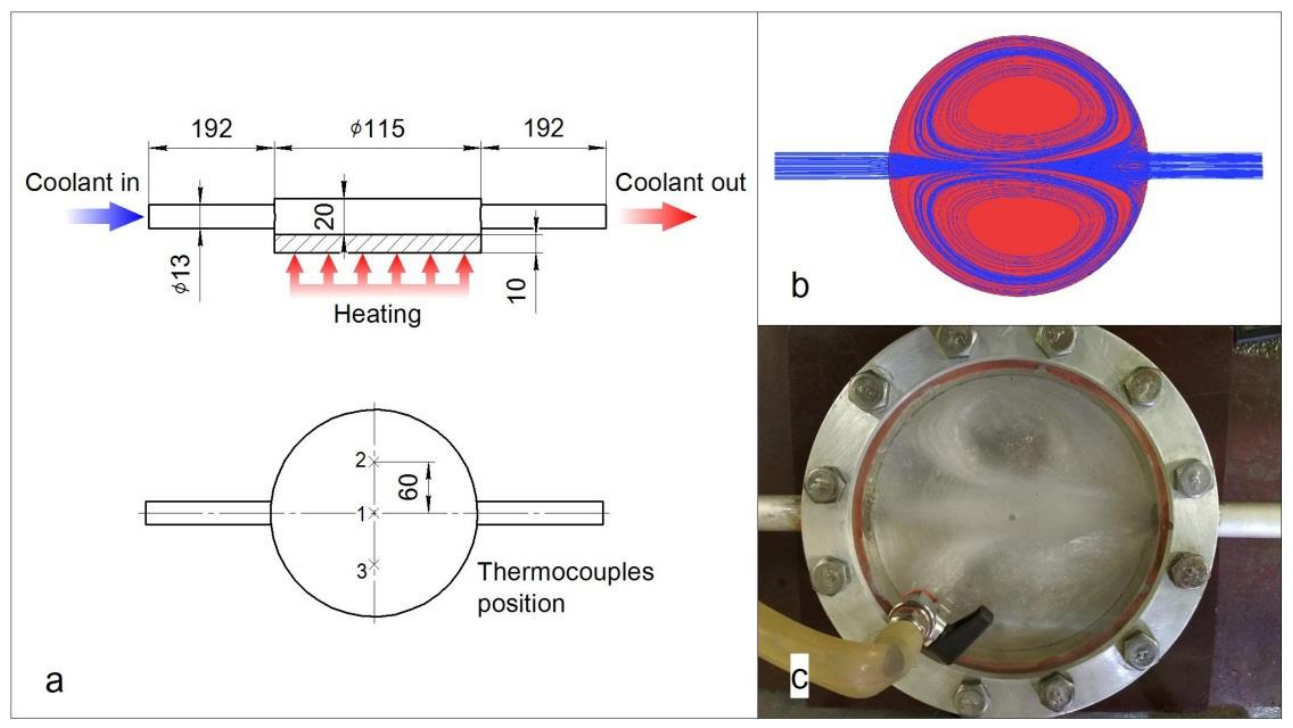

Figure 1. The test duct. $\mathbf{a}-$ General layout; $\mathbf{b}-$ flow pattern in the test duct (simulation resurlts); $\mathbf{c}-$ visualization of the flow in the test duct 


\subsubsection{CFD simulation setup}

The computational domain for CFD simulation was constructed with hexahedral mesh. Several grids with different sizes of mesh cells in whole domain and boundary layer were tested to ensure grid independence. With Enhanced Wall Treatment enabled in $k-\varepsilon$ turbulence model there is no strict requirement for the boundary layer cell size but the general recommendations are to maintain the $\mathrm{y}^{+}$value for the near wall first cell inbetween 30 to 200 , so that the first cell centroid will lie in the logarithmic turbulent layer.

\section{Results and discussion}

Results of the computer simulation are presented at Figures 1-4 in the form of boiling curves compared with experimental data (points on graph). The data is taken from two measurement points at the wall (see Figure 1).

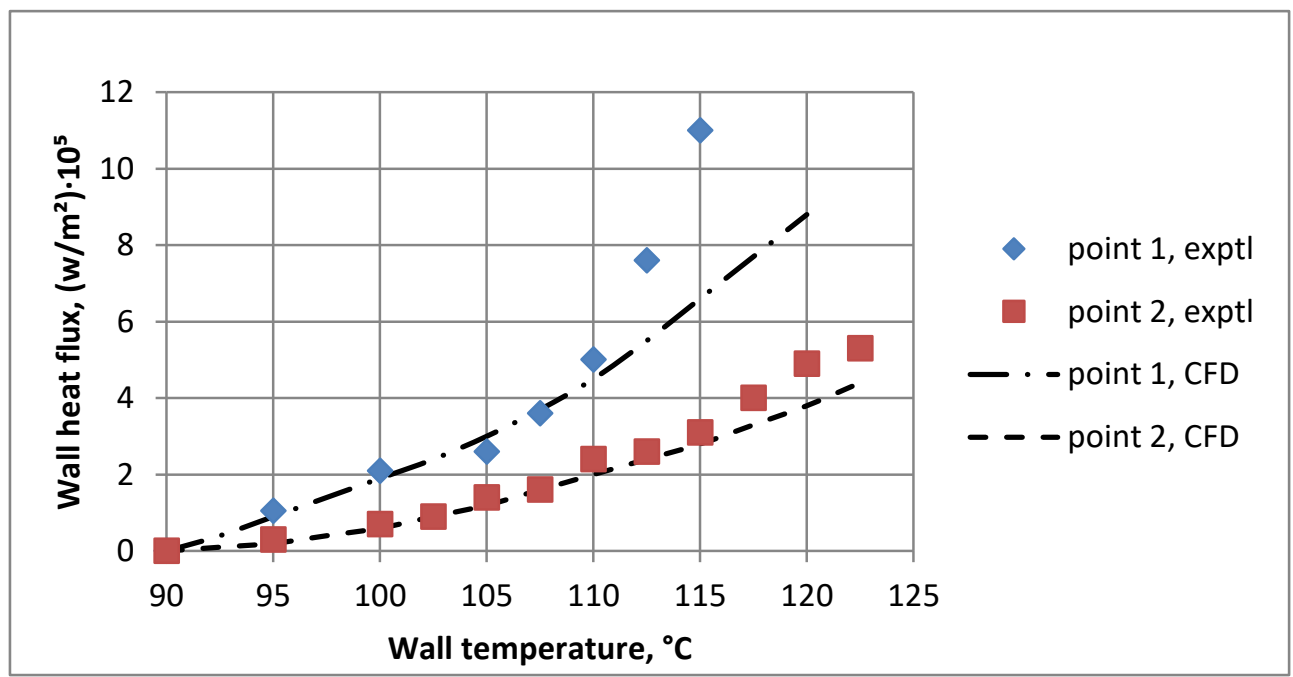

Figure 2. Heat flux versus wall temperature. Water.

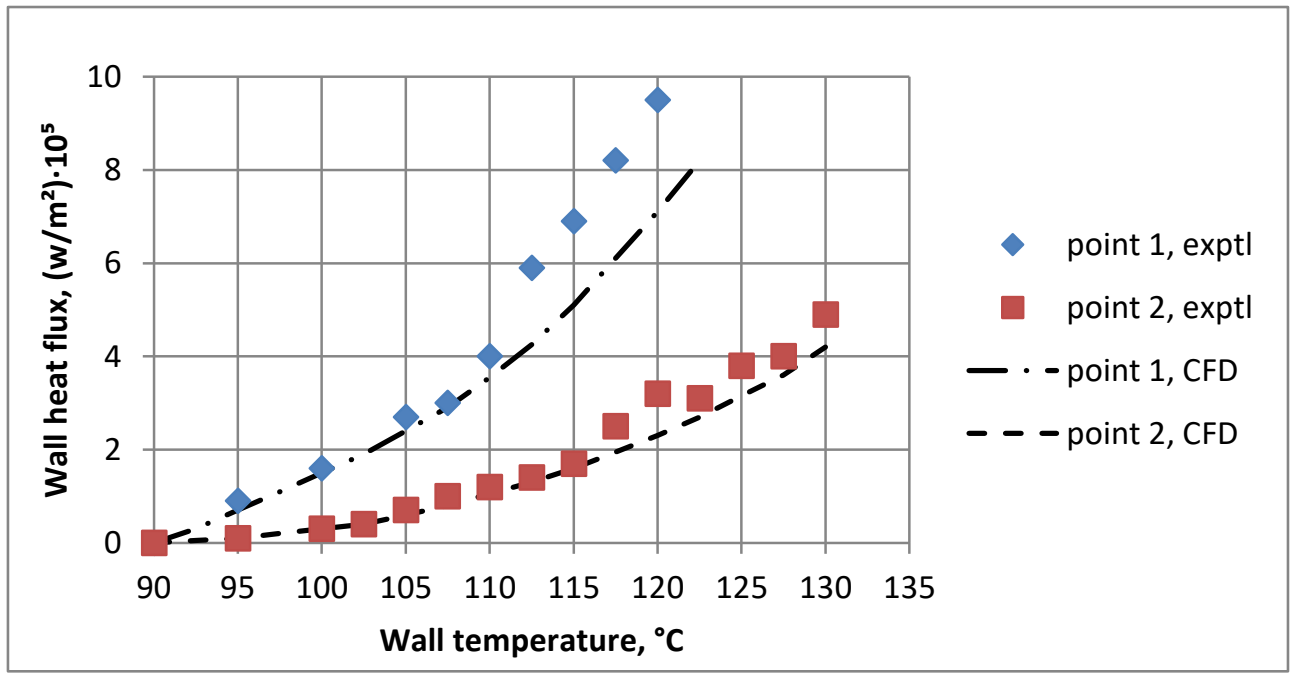

Figure 3. Heat flux versus wall temperature. 50-50 Water-EG. 
The graphs show that in stagnant zones much lower rate of heat transfer is seen and it is close to pool boiling with no rapid transition from pure convection to boiling. The effect of the heat transfer augmentation due to boiling is more pronounced with water as a coolant. In all cases the clear underpredictment of the boiling heat flux is seen for the CFD model, more pronounced in the case of high velocity. The reasons for this deviation could be:

- No account was made for the surface microstructure and the effect of surface "aging" caused by the sedimentation of deposits from the coolant [20]

- The model used in calculations was set with the default parameters and needs an adjustment to be able to give better results.

Nevertheless the model shows decent predictive capability with the maximum error within $5-10 \mathrm{~K}$ in terms of wall temperature and can be used as an instrument to avoid unrealisticaly high wall temperatures in standart CFD simulations for boiling flows.

\section{Conclusions}

The mechanistic wall boiling model is implemented in the Eulerian multiphase model framework available in ANSYS Fluent to simulate subcooled nucleate boiling. The experimental data from author's previous work is used for validation of the model. The following results are obtained:

1. The boiling model is capable of catching the effect of the augmentation of heat transfer caused by nucleate boiling. The degree of accuracy provided by the model is sufficient in terms of wall temperature, so that the model can be used in CFD predictions for IC engine cooling.

2. The model shows clear underprediction of boiling heat transfer, the discrepancy is higher at higher heat fluxes. The origins of this discrepancy can lie in both experimental and numerical simulation fields.

3. The model has a complex structure, consisting of several sub-models and including additional correlations, mostly developed for high pressure conditions. Full adaptation of high pressure models to meet the requirements of low pressure applications is the ultimate goal that has yet to be acchieved.

\section{References}

1. O. Abyzov, Y. Galyshev, A. Shabanov, Dvigatelestroyenie, 2(256), 8-10 (2014)

2. V. Dhir, Annu. Rev. of Fluid Mech., 30(1), 365-401 (1998)

3. G. Son, V. K. Dhir, ASME J. Heat Transfer, 120(1), 183-192 (1998)

4. D. Yuan, L. Pan, D. Chen, 7th Int. Conf. of Mult. Flow, Tampa, FL, 1-10 (2010)

5. S. Kandlikar, ASME Transactions, 120, 388 - 394 (1998)

6. Mc. W. Adams, W. Kennel, C. Minden, R. Carl, P. Picornell, J. Dew, Ind. and Eng. Chem., 41, 1945-1953 (1949)

7. V. Prodanovic, D. Fraser, M. Salcudean, Int. J. Heat Mass Transfer, 45(24), 47274738 (2002)

8. J. C. Chen, Ind. Eng. Chem. Process Des. Dev., 5(3), 322-329 (1966)

9. W. Rohsenow, Trans. ASME, 74, 969 (1952)

10. M. M. Shah, ASHRAE Trans., 83(Pt. I), 205-217 (1977) 
11. K. Robinson, J. G. Hawley, N. A. F. Campbell, Proc. Inst. Mech. Eng., Part D, 217, 877-889 (2004)

12. H. Steiner, A. Kobor, L. A. Gebhard, Int. J. Heat Mass Transfer, 48(19-20), 41614173 (2005)

13. N. Basu, G. R. Warrier, V. K. Dhir, ASME J. Heat Transfer, 127(2), 131-140 (2005)

14. H. Punekar, S. Das, SAE Technical Paper, 2013-01-1651 (2013)

15. N. Kurul, M. Podowski, 27th Nat. Heat Transf. Conf., 30-40 (1991)

16. H. Li, S. Vasquez, H. Punekar, R. Muralikrishnan, Proc. of the ASME 2011 Int. Mech. Eng. Congr. \& Exp. IMECE2011, Paper No. IMECE2011-65539 (2011)

17. J. Tu, G. Yeoh, Int. J. of Heat and Mass Transfer, 45, 1197-1209 (2002)

18. F. White, G. Christoph, Technical Report AFFDL-TR-70-133 (1971)

19. O. Abyzov, Y. Galyshev, A. Zaitsev, A. Sidorov, Izvestiya of International Academy Agricultural education, 35, 7-12 (2017)

20. K. Robinson, J. Hawley, Proc. Instn Mech. Engrs, V.217 Part D: Automobile Engineering (2004) 